\title{
Uncertain Nonlinear Chaotic Gyros Synchronization by Using Adaptive Fuzzy Control
}

\author{
http://dx.doi.org/10.3991/ijoe.v9i3.2601 \\ H. Liu ${ }^{1}$, B. Hou ${ }^{2}$ and W. Xiang ${ }^{1}$ \\ ${ }^{1}$ Huainan Normal University, Huainan, China \\ ${ }^{2}$ North China University of Water Resources and Electric Power, Zhengzhou, China
}

\begin{abstract}
In this paper, the control problem of synchronizing two identical chaotic gyros systems in the presence of system uncertainties is addressed by using adaptive fuzzy sliding mode control, while the structure of the gyros and the bounds of the system uncertainties are entirely unknown. The controller can guarantee a fast convergence of the synchronization errors and all of the signals in the closed-loop system remain bounded. Finally simulation results are provided to illustrate the effectiveness of the proposed control method.
\end{abstract}

Index Terms-Adaptive Fuzzy Control, Chaotic Gyros, Synchronization

\section{INTRODUCTION}

Gyros are an interesting kind of nonlinear systems that have attracted many researchers during the last two decades due to their potential applications in the aeronautical, navigational and space engineering domains. Recently, much research has identified various types of gyro systems with linear or nonlinear damping characteristics. These systems have a diverse range of dynamic behavior including both chaotic motions and sub-harmonic. [1-3].

During the past decade, adaptive fuzzy control systems designs have been extensively studied [4-6]. The main idea of adaptive fuzzy control is as follows. Based on the universal approximation theorem [7], a fuzzy model to describe the input and output behavior of the controlled system is firstly constructed. Then a controller is designed based on the fuzzy model and adaptive laws are derived to update the parameters of the fuzzy systems. And many approaches for achieving chaos synchronization using fuzzy systems have been proposed [8].

This paper will develop a fuzzy sliding mode control scheme to achieve the synchronization of two chaotic gyro systems with different initial conditions, system uncertainties and external disturbances.

\section{PROBlem Description}

The symmetric gyroscope mounted on a vibrating base can be seen in Fig.1.

The dynamics of the gyro system with linear-plus-cubic damping of the angel $\theta$ can be described as [8]:

$$
\begin{aligned}
& \ddot{\theta}+\alpha \frac{(1-\cos \theta)^{2}}{\sin ^{3} \theta}-\beta \sin \theta+c_{1} \dot{\theta}+c_{2} \dot{\theta}^{3} \\
& =f \sin \omega t \sin \theta
\end{aligned}
$$

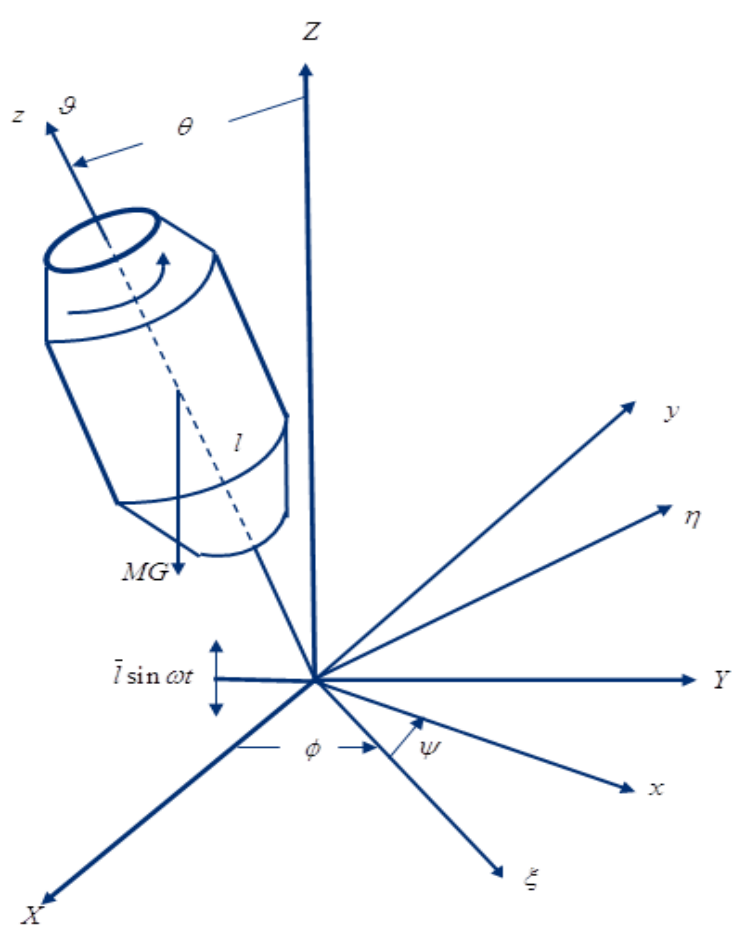

Figure 1. A schematic diagram of a symmetric gyroscope.

where $f \sin \omega t$ is a parametric excitation, $c_{1} \dot{\theta}$ and $c_{2} \dot{\theta}^{3}$ represent linear and nonlinear damping terms, respectively. $\alpha \frac{(1-\cos \theta)^{2}}{\sin ^{3} \theta}-\beta \sin \theta$ is a nonlinear resilience force. Let $x_{1}=\theta, x_{2}=\dot{\theta} \quad$ and $g(\theta)=\alpha \frac{(1-\cos \theta)^{2}}{\sin ^{3} \theta}-\beta \sin \theta$, system (1) can be rewritten as:

$$
\begin{aligned}
& \dot{x}_{1}=x_{2}, \\
& \dot{x}_{2}=g\left(x_{1}\right)-c_{1} x_{2}-c_{2} x_{2}^{3}+(\beta+f \sin \omega t) \sin x_{1} .
\end{aligned}
$$

This gyro system (1) or (2) exhibits chaotic dynamics when $f=35, \alpha=10, \beta=1, c_{1}=0.5, c_{3}=0.05$ and $\omega=2$. The chaotic behavior is shown in Fig.2-Fig.4. with the initial values $x_{1}=0, x_{2}=0$. 


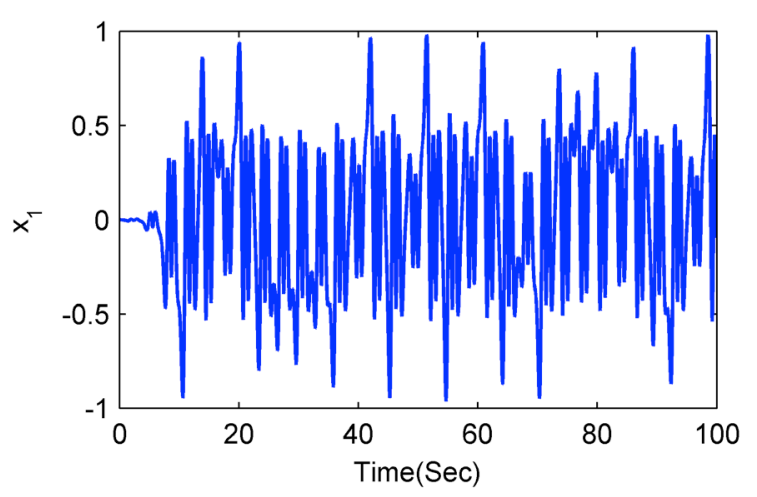

Figure 2. The response of $x_{1}$.

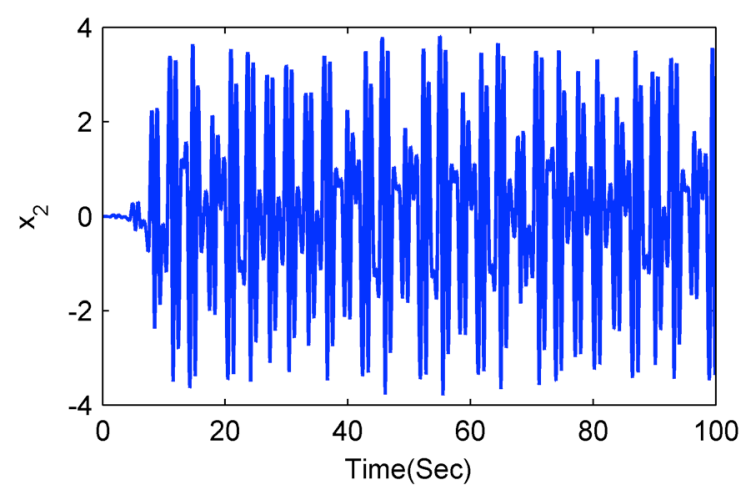

Figure 3. The response of $x_{2}$.

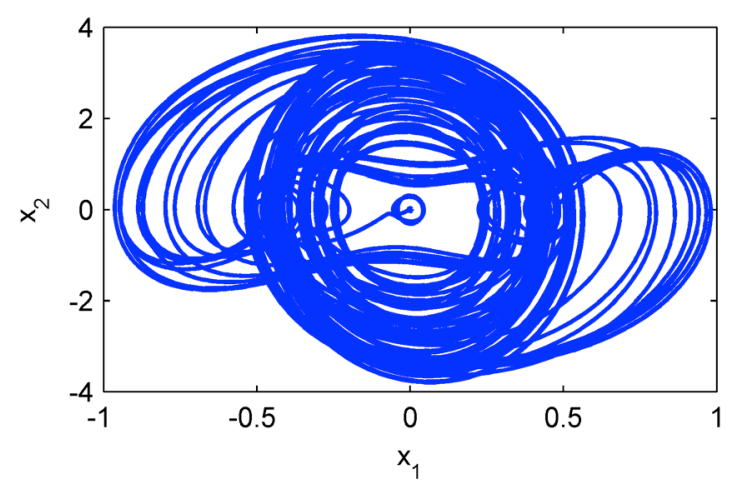

Figure 4. Chaotic attractor of gyro system.

The fuzzy logic system that employs singleton fuzzification, sum-product inference and center-off-sets defuzzification can be modeled by [7]

$$
\alpha(x)=\frac{\sum_{j=1}^{N} \theta_{j} \Pi_{i=1}^{n} \mu_{F_{i}^{j}}\left(x_{i}\right)}{\sum_{j=1}^{N}\left[\Pi_{i=1}^{n} \mu_{F_{i}^{j}}\left(x_{i}\right)\right]},
$$

where $\alpha(x)$ is the output of the fuzzy system, $x$ is the input vector, $\mu_{F_{i}^{j}}\left(x_{i}\right)$ is $x_{i}{ }^{\prime} s$ membership of $j$ th rule and $\theta_{j}$ is the centroid of the $j$ th consequent set. Eq. (2) can be rewritten as:

$$
\alpha(x)=\vartheta^{T} \psi(x)
$$

with $\vartheta=\left[\vartheta_{1}, \mathrm{~L}, \vartheta_{N}\right]^{T}, \psi(x)=\left[p_{1}(x), p_{2}(x), \mathrm{L}, p_{N}(x)\right]^{T}$, and the fuzzy basis function can be expressed as: $p_{j}(x)=\frac{\prod_{i=1}^{n} \mu_{F_{i}^{j}}\left(x_{i}\right)}{\sum_{j=1}^{N}\left[\Pi_{i=1}^{n} \mu_{F_{i}^{j}}\left(x_{i}\right)\right]}$.

\section{SyNCHRONIZATION PROBLEM FORMULATION}

Consider two chaotic gyro systems of the form (2) and:

$$
\begin{aligned}
\dot{y}_{1} & =y_{2}, \\
\dot{y}_{2} & =g\left(y_{1}\right)-c_{1} y_{2}-c_{2} y_{2}^{3}+\beta \sin y_{1} \\
& +f \sin \omega t \sin x_{1}+\Delta f\left(y_{1}, y_{2}\right)+u .
\end{aligned}
$$

as the master and slave chaotic systems, respectively. $u \in R$ is the control input, and $\Delta f\left(y_{1}, y_{2}\right)$ represents system uncertainties and external disturbances. In this paper, we assume that $\Delta f\left(y_{1}, y_{2}\right)$ is bounded, i.e., there exists some constant $a>0$ satisfies $\left|\Delta f\left(y_{1}, y_{2}\right)\right| \leq a$, but the value of $a$ is entirely unknown.

The objective of this paper is to design an appropriate control inpeut such that for any initial conditions of the two chaotic systems (2) and (5), the states of the slave system converges to that of the master, i.e.

$$
\lim _{t \rightarrow \infty}\|y(t)-x(t)\|=0
$$

where $y(t)=\left[y_{1}, y_{2}\right]^{T}$ and $x(t)=\left[x_{1}, x_{2}\right]^{T}$.

Main Results

To begin with, let us define the synchronization errors between master and slave systems as:

$$
e=y-x=\left[e_{1}, e_{2}\right]^{T},
$$

and the filtered synchronization error as

$$
s=\left[\frac{d}{d t}+\lambda\right] e_{1}=\lambda e_{1}+e_{2},
$$

where $\lambda>0$ is a control design constant, and the polynomial $H(s)=\lambda^{n-1}+(n-1) \lambda^{n-2} s+\mathrm{L}+s^{n-1}$ is Hurwitz.

Then, the synchronization error dynamic can be expressed as

$$
\begin{aligned}
\dot{e}_{1} & =e_{2}, \\
\dot{e}_{2} & =-c_{1} e_{2}+g\left(y_{1}\right)-g\left(x_{1}\right)+c_{2}\left(x_{2}^{3}-y_{2}^{3}\right) \\
& +(\beta+f \sin \omega t)\left(\sin y_{1}-\sin x_{1}\right) \\
& +\Delta f\left(y_{1}, y_{2}\right)+u .
\end{aligned}
$$

Since $\Delta f\left(y_{1}, y_{2}\right)$ is unknown, then it can be approximated, through the fuzzy logic system (7), by

$$
\Delta f\left(y_{1}, y_{2}\right)=\vartheta^{T} \psi(x, y)+\delta
$$

where $\delta$ is the approximation error of the fuzzy logic systems. As the same analysis in $[4,5]$, there has a positive constant $\varepsilon>0$ such that $|\delta|<\varepsilon$. Then we can obtain the following inequality easily: 


$$
\begin{gathered}
s \Delta f\left(y_{1}, y_{2}\right)=s \vartheta^{T} \psi(x, y)+s \delta \\
\leq s \frac{\|\vartheta\| \psi^{T}(x, y) \psi(x, y)}{l\|\vartheta\|} l+s \delta \\
\leq \frac{1}{2 l^{2}} s^{2}\|\vartheta\|^{2} \psi^{T}(x, y) \psi(x, y) \\
\quad+\frac{1}{2} l^{2}+\frac{1}{2} s^{2}+\frac{1}{2} \varepsilon^{2},
\end{gathered}
$$

where $l$ is a positive constant.

Then, the control input $u$ can be designed as

$$
\begin{aligned}
u= & c_{1} e_{2}-g\left(y_{1}\right)+g\left(x_{1}\right)-c_{2}\left(x_{2}^{3}-y_{2}^{3}\right) \\
& -(\beta+f \sin \omega t)\left(\sin y_{1}-\sin x_{1}\right) \\
& -\frac{1}{2 l^{2}} s^{2} \hat{\tau} \psi^{T} \psi-k s,
\end{aligned}
$$

where $k>0$ is design parameter, and its value will be define later. $\hat{\tau}$ is the estimation of the unknown fuzzy system parameter $\tau=\|\theta\|^{2}$. The adaption law can be designed as

$$
\dot{\hat{\tau}}=\frac{r}{2 l^{2}} s^{2} \psi^{T} \psi-m \hat{\tau},
$$

with $m>0$ is constant.

From above discussions, now we will give the following results:

Theorem 1. Consider the master chaotic system (2) and the slave chaotic system (5). The control law is defined as (12), the parameter of the fuzzy logic system updated by the adaption law (13), then we can achieve the synchronization between (2) and (5) with all signals in the closedloop system remain bounded.

Proof. From (8), we have its derivative with respect to time is

$$
\begin{aligned}
\dot{s}= & -c_{1} e_{2}+g\left(y_{1}\right)-g\left(x_{1}\right)+c_{2}\left(x_{2}^{3}-y_{2}^{3}\right) \\
& +(\beta+f \sin \omega t)\left(\sin y_{1}-\sin x_{1}\right) \\
& +\Delta f\left(y_{1}, y_{2}\right)+u+\lambda e_{2} .
\end{aligned}
$$

Substituting (12) into (14) gives

$$
\dot{s}=\Delta f\left(y_{1}, y_{2}\right)-\frac{1}{2 l^{2}} s^{2} \hat{\tau} \psi^{T} \psi-k s .
$$

Then we have

$$
\begin{aligned}
s \dot{s} & =-\left(k-\frac{1}{2}\right) s^{2}-\frac{1}{2 l^{2}} s^{2} \tilde{\boldsymbol{\tau}} \psi^{T}(x, y) \psi(x, y) \\
& +\frac{1}{2} l^{2}+\frac{1}{2} \varepsilon^{2} .
\end{aligned}
$$

where $\tilde{\tau}=\hat{\tau}-\tau$ is the estimation error of the fuzzy parameter.
Let us define the Lypunov function candidate as

$$
V=\frac{1}{2} s^{2}+\frac{1}{2 r} \tilde{\tau}^{2}
$$

Then we can obtain

$$
\dot{V} \leq\left(-k+\frac{1}{2}\right) s^{2}-\frac{m}{2 r} \tilde{\tau}^{2}+\frac{1}{2} l^{2}+\frac{1}{2} \varepsilon^{2}+\frac{1}{2} \tau^{2}
$$

If we can choose $k>\frac{1}{2}$, and let $a_{0}=\min \left\{2\left(k-\frac{1}{2}\right), m\right\}, b_{0}=\frac{1}{2} l^{2}+\frac{1}{2} \varepsilon^{2}+\frac{1}{2} \tau^{2}$, then

can be rewritten as:

$$
\dot{V} \leq a_{0} V+b_{0},
$$

After some straightforward manipulators, we can obtain

$$
V(t) \leq\left(V\left(t_{0}\right)-\frac{b_{0}}{a_{0}}\right) e^{-a_{0}\left(t-t_{0}\right)}+\frac{b_{0}}{a_{0}}, \forall t \geq t_{0} .
$$

As a result, all signals in the closed-loop system will remain bounded, and the synchronization error will converge to an adjustable region of the origin. This ends the proof of Theorem 1 .

\section{Simulation STUdies}

The fuzzy logic system uses $x, y$ as the inputs. For each variable of $x$ and $y$, we define five Gaussian membership functions uniformly distributed on the interval $[-10,10]$. The Gaussian membership functions can be seen in Fig.5.

The initial values of the two chaotic system are chosen as $x(0)=[1.6,0]^{T}$ and $y(0)=[-3,3]^{T}$. And the control design parameters are chosen as $\lambda=3, r=100$.

The simulation results are shown in Fig.6-Fig.9. From the results we can conclude that good synchronization performance has been achieved, and the synchronization errors have fast convergence. Fig.9 shows the smoothness of the control inputs.

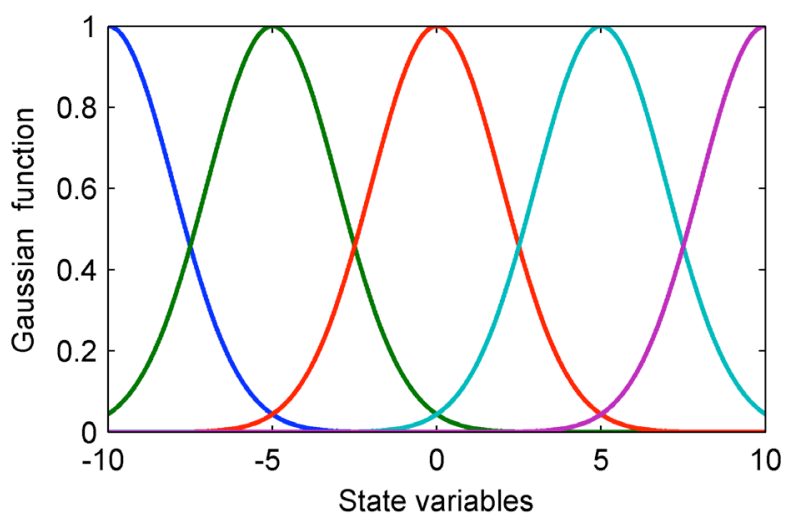

Figure 5. Gaussian membership functions. 


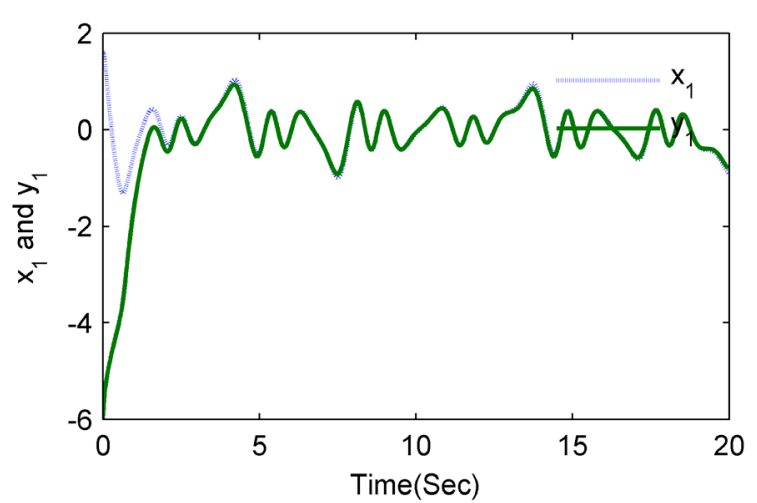

Figure 6. Response of $x_{1}$ and $y_{1}$.

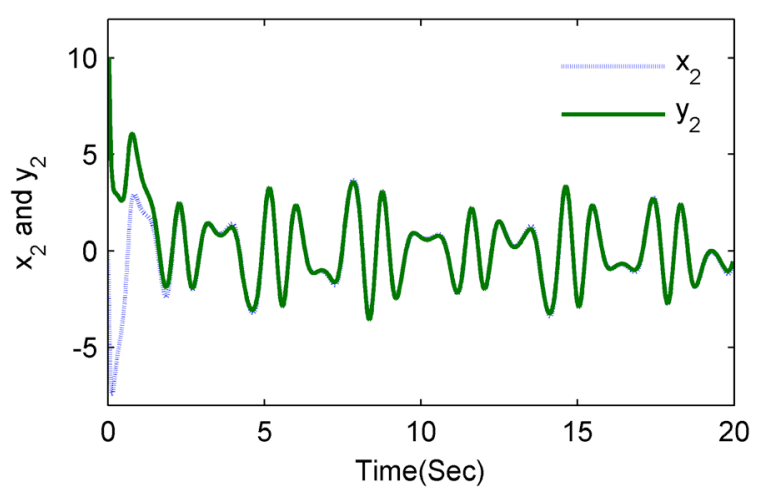

Figure 7. Response of $x_{2}$ and $y_{2}$.

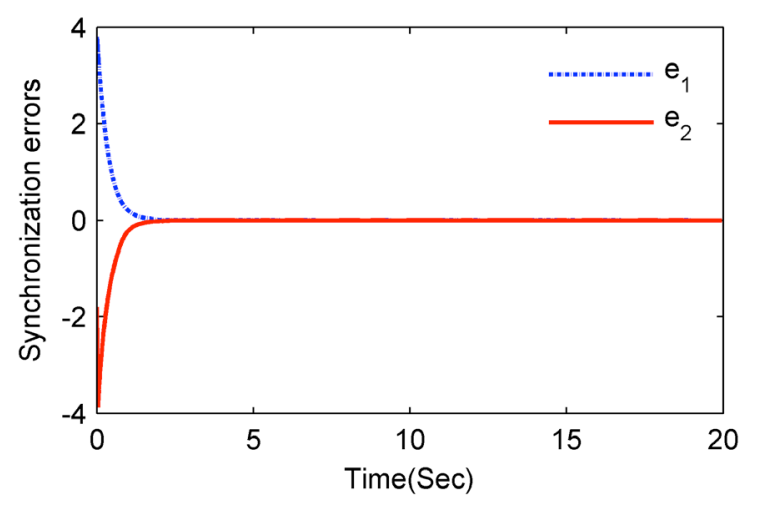

Figure 8. Response of synchronization errors.

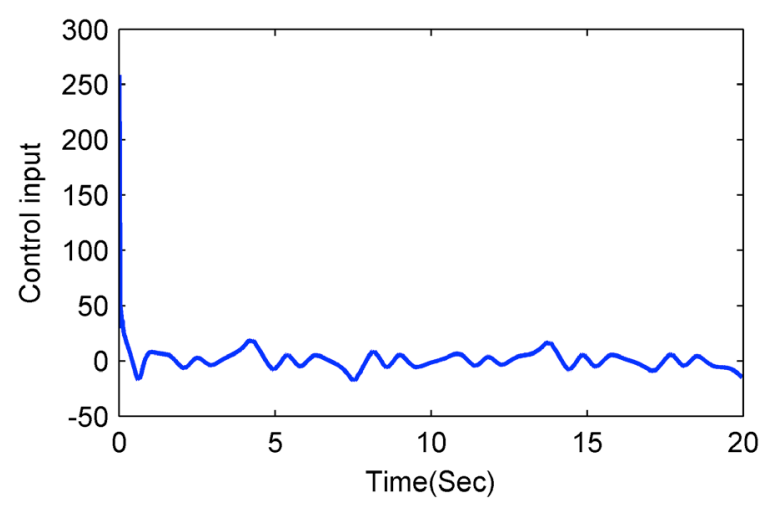

Figure 9. Response of the control input.

\section{CONCLUSiOnS}

In this paper, the synchronization between two identical chaotic gyros systems is addressed by means of adaptive fuzzy sliding mode control method. Compared with other methods proposed in the literature for the synchronization of chaotic systems, the main contribution of this paper consists in that no prior knowledge of the structure of the gyros and the bounds of the system uncertainties is required in the controller design. Furthermore, the proposed method does not require information about the parameters of the system, and this can make it easy to use in practical applications.

\section{REFERENCES}

[1] M. Roopaei, M.Z. Jahromi, R. Johu, T.C. Lin, "Unknown nonlinear chaotic gyros synchronization using adaptive fuzzy sliding mode control with unknown dead-zone input", Commun Nonlinear Sci Numer Simulat, vol. 15, pp. 2536-2545, 2010. http://dx.doi.org/10.1016/j.cnsns.2009.09.022

[2] R.V. Dooren, "Comments on chaos and chaos synchronization of a symmetric gyro with linear-plus-cubic damping", J. Sound Vibr, vol. 268, pp. 632-634, 2003. http://dx.doi.org/10.1016/S0022460X(03)00343-2

[3] Z.M. Ge, H.K. Chen, "Bifurcations and chaos in a rate gyro with harmonic excitation", J. Sound Vibr, vol. 194, pp. 107-117, 1996. http://dx.doi.org/10.1006/jsvi.1996.0348

[4] H. Liu, H.J. Yu, W. Xiang, "Adaptive fuzzy nonlinear inversionbased control for uncertain chaotic systems.", Chinese physics $B$, vol. 21, pp. 1205051, 2005.

[5] A. Boulkroune, M. Tadjine, M. M'Saad, M. Farza, "Fuzzy adaptive controller for MIMO nonlinear systems with known and unknown direction.” Fuzzy Sets and Systems, vol.161, pp. 797-820, 2010. http://dx.doi.org/10.1016/j.fss.2009.04.011

[6] J.P. Yu, B. Chen, H.S. Yu, J.W. Gao, “Adaptive fuzzy tracking control for the permanent magnet synchronous motor drive system via backstepping", Nonlinear Analysis: Real World Applications, vol. 12, pp. 671-681, 2011. http://dx.doi.org/10.1016/j.nonrwa. 2010.07.009

[7] Wang L X, "Adaptive fuzzy systems and control: Design and stabilitiy analysis", New Jersey: Prentice Hall, 1994.

[8] H.T. Yau, "Chaos synchronization of two uncertain chaotic nonlinear gyros using fuzzy sliding mode control", Mechanical Systems and Signal Processing, vol. 22, pp. 408-418, 2008. http://dx.doi.org/10.1016/j.ymssp.2007.08.007

\section{AUTHORS}

H. Liu received his M.S. degree in applied mathematics from Xinan Jiaotong University, Chengdu, China in 2006. $\mathrm{He}$ is now a lecture in the departments of mathematics and computational science, Huainan Normal University. His main Research interest is nonlinear system control and fuzzy control.

B. Hou received his M.S. degree in Applied Mathematics, Xinan Jiaotong University, Chengdu, China in 2006. He is now a lecture in the Department of mathematics and Information Science, North China University of Water Resources and Electric Power. His main Research interest is nonlinear system control and adaptive control.

W. Xiang received his Ph.D. degree in General and Fundamental Mechanics, Nanjing University of Aeronautics and Astronautics, in 2010 . He is now a lecture in the departments of mathematics and computational science, Huainan Normal University. His main Research interest is nonlinear system contro, sliding mode control and adaptive control.

Received 19 March 2013. Published as resubmitted by the authors 12 June 2013. 